\title{
Geometric Properties of Boundary Orbit Accumulation Points 12
}

\author{
Steven G. Krantz 3
}

November 23, 2018

Dedicated to Leon Ehrenpreis, a fine mathematician and a wonderful human being.

\begin{abstract}
We study the automorphism group action on a bounded domain in $\mathbb{C}^{n}$. In particular, we consider boundary orbit accumulation points, and what geometric properties they must have. These properties are formulated in the language of Levi geometry.
\end{abstract}

\footnotetext{
${ }^{1}$ Key Words: orbit accumulation point, pseudoconvexity, orbit, holomorphic mapping.

${ }^{2}$ MR Classification Numbers: 32M05, 32M17, 32T17.

${ }^{3}$ Author supported in part by the National Science Foundation and by the Dean of the Graduate School at Washington University.
} 


\section{Introduction}

In this paper a domain $\Omega \beta \mathbb{C}^{n}$ is a connected open set. We let $\mathcal{O}(\Omega)$ denote the algebra of holomorphic functions on $\Omega$. Also we let $\operatorname{Aut}(\Omega)$ be the group (under composition of mappings) of biholomorphic self-maps of $\Omega$. The standard topology on $\operatorname{Aut}(\Omega)$ is that of uniform convergence on compact sets (equivalently, the compact-open topology).

We shall use the following notation: $D$ denotes the unit disc in the complex plane. We let $D^{2}=D \times D$ denote the bidisc, and $D^{n}=D \times D \times \cdots \times D$ the polydisc in $\mathbb{C}^{n}$. The symbol $B=B_{n}$ is the unit ball in $\mathbb{C}^{n}$.

Certainly domains with transitive automorphism group are of some interest. But they are relatively few in number (see the classification theory of Cartan, as described in [HEL]). A very natural and compelling alternative is to study domains with noncompact automorphism group. A bounded domain $\Omega$ has noncompact automorphism group if there is a sequence $\varphi_{j} \in \operatorname{Aut}(\Omega)$ such that no subsequence converges uniformly on compact sets to another automorphism. Obversely, the automorphism group is compact if every sequence $\left\{\varphi_{j}\right\}$ in $\operatorname{Aut}(\Omega)$ has a subsequence that converges uniformly on compact sets to another automorphism. In this regard, the following result of $H$. Cartan is central and useful (see $[\mathrm{NAR}]$ ):

Theorem 0.1 Let $\Omega \beta \mathbb{C}^{n}$ be a bounded domain. Then $\Omega$ has noncompact automorphism group if and only if there are a point $P \in \partial \Omega$ and a point $X \in \Omega$ and automorphisms $\varphi_{j} \in \operatorname{Aut}(\Omega)$ such that $\lim _{j \rightarrow \infty} \varphi_{j}(X)=P$.

A point $P \in \partial \Omega$ is called a boundary orbit accumulation point if there is a point $X \in \Omega$ and a sequence $\varphi_{j} \in \operatorname{Aut}(\Omega)$ such that $\lim _{j \rightarrow \infty} \varphi_{j}(X)=P$. Of special interest is the case when there is a single automorphism $\psi$ such that $\lim _{j \rightarrow \infty} \psi^{j}(X)=P$. (Here $\psi^{j}$ denotes the composition of $\psi$ with itself $j$ times when $j=0,1,2, \ldots$; also, if $j<0$, then $\psi^{j}$ denotes the composition of $\psi^{-1}$ with itself $|j|$ times.) In this latter circumstance we call $P$ a special boundary orbit accumulation point. It is not clear when an arbitrary boundary orbit accumulation point is a special boundary orbit accumulation point.

We shall use Section 1 to collect some simple, preliminary results that have independent interest. 


\section{Background Results}

The result that inspires the present work comes from [GRK1]:

Theorem 1.1 Let $\Omega \beta \mathbb{C}^{n}$ be a smoothly bounded domain. Suppose that $P \in \partial \Omega$ is a boundary orbit accumulation point. Then $P$ is a point of Levi pseudoconvexity.

There is an analogous result for domains without smooth boundary (and in which the conclusion involves Hartogs pseudoconvexity). But we shall have no use for it in the present paper. See [GRK1] for the details.

We first give an example to emphasize that, even though the boundary orbit accumulation point is pseudoconvex, nearby points need not be.

EXAMPLE 1.2 Let $B \beta \mathbb{C}^{n}$ be the unit ball with defining function $\rho(z)=$ $|z|^{2}-1$ (see [KRA1] for the concept of definining function). Let $\phi$ be a $C_{c}^{\infty}$ function on $\mathbb{C}^{n}$ with these properties:

(a) $\phi$ is real-valued and $0 \leq \phi(z) \leq 1 / 10$ for all $z \in \mathbb{C}^{n}$.

(b) $\phi$ is radial about the point $(i, 0)$.

(c) $\operatorname{supp} \phi ß B((i, 0), 1 / 10)$.

(d) $\phi(z)=1 / 10$ for $|z-(i, 0)|<1 / 20$.

Set

$$
\Omega^{\prime}=\left\{z \in \mathbb{C}^{2}:-1+|z|^{2}+\phi(z)<0\right\}
$$

and

$$
\Omega=\bigcap_{j=-\infty}^{\infty} \Phi_{1 / 2}^{j}\left(\Omega^{\prime}\right)
$$

where

$$
\Phi_{a}\left(z_{1}, z_{2}\right)=\left(\frac{z_{1}-a}{1-\bar{a} z_{1}}, \frac{\sqrt{1-|a|^{2}} z_{2}}{1-\bar{a} z_{1}}\right),
$$

any $a \in \mathbb{C},|a|<1$. It is easy to check, by direct calculation, that $\Phi_{a}$ is an automorphism of the unit ball $B \beta \mathbb{C}^{n}$. And the domain $\Omega$ will be the unit ball with infinitely many strongly pseudoconcave dents that accumulate at the points $(1,0)$ and $(-1,0)$. 
Now it is plain that the point $(1,0) \in \partial \Omega$ is a boundary orbit accumulation point. In fact we may let $X=(0,0)$ and $\varphi_{j}(z)=\Phi_{1 / 2}^{-j}(z)$ for $j=1,2, \ldots$ So $(1,0) \in \partial \Omega$ is certainly pseudoconvex. Notice that, at points along the normal line through $(i, 0),-1+|z|^{2}+\phi(z)$ is negative when $z$ is at least $1 / 5$ units from the boundary of the ball $B$. And $-1+|z|^{2}+\phi(z)$ is positive at $(i, 0)$. So there must be an intermediate point $\widetilde{z}$ on this line segment - a point inside the unit ball $B$-where $-1+|z|^{2}+\phi(z)$ vanishes. It follows that $\widetilde{z}$ is a boundary point of $\Omega^{\prime}$. Hence $\Phi_{1 / 2}^{j}(\widetilde{z})$ is a boundary point for each $j$. The boundary points $\Phi_{1 / 2}^{j}(\widetilde{z})$ will be strictly pseudoconcave.

It must be noted that, in the last example, $\Omega$ does not have smooth boundary. In fact, at the boundary points $(1,0),(-1,0)$, the boundary is only Lipschitz.

Of course the disc $D ß \mathbb{C}$ has noncompact automorphism group. Let

$$
\varphi_{a}(\zeta)=\frac{\zeta-a}{1-\bar{a} \zeta}
$$

for $a \in \mathbb{C},|a|<1$. Then the automorphisms

$$
\left\{\frac{\zeta-(1-1 / j)}{1-(1-1 / j) \zeta}: j \in \mathbb{Z}\right\}
$$

are a sequence of automorphisms of $D$ that have no subsequence converging to an automorphism. Indeed, any subsequence either converges to the constant function 1 or the constant function -1 . It is a fact - see [KRA2]that any domain in $\mathbb{C}$ having $C^{1}$ boundary and noncompact automorphism group must be conformally equivalent to the disc. This is true without any topological hypotheses on the domain! In $\mathbb{C}^{n}$, the first correct theorem of this nature - due to Bun Wong [WON] and Rosay [ROS] - is that any $C^{2}$ bounded domain in $\mathbb{C}^{n}$ with a boundary orbit accumulation point that is strongly pseudoconvex must be biholomorphic to the unit ball $B$. It is not known in general which smoothly bounded domains have noncompact automorphism group. Certainly there are finite type domains with non-compact automorphism group-see [BEP1], [BEP2]. More on this matter in what follows.

In this paper we concentrate mainly on bounded domains. But we shall make a few remarks right now about unbounded domains. 
EXAMPLE 1.3 In the complex plane $\mathbb{C}$, there are unbounded domains with noncompact automorphism group that are not the disc. The simplest example is when the domain $\Omega$ is the entire complex plane $\mathbb{C}$. The punctured plane also has this property.

\section{New Results}

The statement of Theorem 1.1 makes it desirable to have a formulation purely in terms of the intrinsic, invariant geometry of the domain. For instance, one might hope to be able to say something about the completeness of the Kobayashi metric at a boundary orbit accumulation point. Unfortunately, the following example dashes that hope:

EXAMPLE 2.1 Let $B \beta \mathbb{C}^{2}$ be the unit ball with definining function $\rho(z)=$ $|z|^{2}-1$. Let $\phi$ be a $C_{c}^{\infty}$ function on $\mathbb{C}^{n}$ with these properties:

(a) $\phi$ is real-valued and $0 \leq \phi(z) \leq 1 / 10$ for all $z \in \mathbb{C}^{n}$.

(b) $\phi$ is radial about the point $(i, 0)$.

(c) $\operatorname{supp} \phi ß B((i, 0), 1 / 10)$.

(d) $\phi(z)=1 / 10$ for $|z-(i, 0)|<1 / 20$.

Set

$$
\Omega^{\prime}=\left\{z \in \mathbb{C}^{2}:-1+|z|^{2}+\phi(z)<0\right\}
$$

and

$$
\Omega=\bigcap_{-1<a<1} \Phi_{a}\left(\Omega^{\prime}\right)
$$

where

$$
\Phi_{a}\left(z_{1}, z_{2}\right)=\left(\frac{z_{1}-a}{1-\bar{a} z_{1}}, \frac{\sqrt{1-|a|^{2}} z_{2}}{1-\bar{a} z_{1}}\right),
$$

any $a \in \mathbb{C},|a|<1$. Then $\Omega$ is the unit ball with a groove stretching from $(-1,0)$ to $(1,0)$. This new domain is strongly pseudoconcave along an entire curve from $(-1,0)$ to $(1,0)$. Of course the point $(1,0)$ is still a boundary orbit accumulation point. Indeed the automorphisms $\Phi_{a},-1<a<0$, send $(0,0)$ to $(1,0)$. And, along the curve $\gamma(t)=(t, 0), 0<t<1$, the Kobayashi distance to the boundary point is infinite. 
Now write $\Phi_{a}(z)=\left(\varphi_{a}^{1}(z), \varphi_{a}^{2}(z)\right)$. Let the point $\widetilde{z}$ be as at the end of Example 1.2. We consider, in the same domain $\Omega^{\prime}$ as above, the curve

$$
t \longmapsto\left(\left[1-(1-t)^{4}\right] \Phi_{t}(\widetilde{z}), 0\right) \quad, \quad 0 \leq t \leq 1
$$

Then this curve terminates at $(-1,0)$ and is tangent to the boundary to fourth order at that point. For $Q \in \partial \Omega$, let $\nu_{Q}$ denote the outward Euclidean unit normal vector at $Q$. If we take advantage of the estimate in [KRA3], to the effect that, near a strongly pseudoconcave boundary point $Q$, a point $Q^{*}=Q-\delta \nu_{Q}$ satisfies the estimate

$$
F_{K}^{\Omega}\left(Q^{*}, \nu\right) \approx \delta^{-3 / 4}
$$

and moreover, in the tangential direction,

$$
F_{K}^{\Omega}\left(Q^{*}, \tau\right) \approx C
$$

then it is easy to see that the distance to the boundary point $(-1,0)$ along the curve $\gamma$ is finite. Therefore, at least on a domain with Lipschitz boundary, it is not the case that a boundary orbit accumulation point will be a point at which the Kobayashi metric is complete.

On the positive side, we can prove the following result:

Proposition 2.2 Let $\Omega ß \mathbb{C}^{2}$ be a smoothly bounded domain and $P \in \partial \Omega$ a boundary orbit accumulation point that is of finite type. Then it is not possible for $\Omega$ to have Levi pseudoconcave boundary points.

Proof: Assume that $P$ is the limit of $\varphi_{j}(X)$ for some point $X \in \Omega$ and some automorphisms $\varphi_{j}$. Since $P$ is of finite type and the complex dimension is 2 , we may use results of [CAT] and [ALA] to see that curves in $\Omega$ terminating at $P$ must have infinite length in the Kobayashi metric.

If $Q$ is a strongly pseudoconcave boundary point, obviously distinct from $P$, then we know from the estimates in [KRA3] that the Kobayashi distance of $Q$ to $X$ is finite. Then the $\varphi_{j}$ applied to $Q$ must move $Q$ into the interior of $\Omega$ (because $X$ is being pushed infinitely far towards $P$ ). Hence $\varphi$ is not an automorphism. That is a contradiction. We conclude that $Q$ cannot exist.

A well-known conjecture in the subject says this: 
The Greene-Krantz Conjecture: Let $\Omega$ be a smoothly bounded domain in $\mathbb{C}^{n}$. If $P \in \partial \Omega$ is a boundary orbit accumulation point, then $P$ is a point of finite type in the sense of Kohn/D'Angelo/Catlin.

This conjecture has not been established in full generality. But results in $[\mathrm{KIM}]$ and [KIK1] support the conjecture. Now we have

Proposition 2.3 Let $\Omega ß \mathbb{C}^{2}$ be a smoothly bounded, pseudoconvex domain. Let $P \in \partial \Omega$ be a boundary orbit accumulation point. Assume that the Greene-Krantz conjecture is true. Then any path ending at $P$ will have infinite length in the Kobayashi metric.

Proof: This result is almost obvious. For the hypothesis implies that $P$ is of finite type. And now the estimates on the Kobayashi metric in [CAT], together with the calculations in [ALA], give the result about infinite length of paths.

\section{A Boundary Orbit Accumulation Point Char- acterization of Domains}

In [ROS] and [WON], for instance, it is shown that if a bounded domain has a strongly pseudoconvex boundary orbit accumulation point then that domain must be biholomorphic to the unit ball in $\mathbb{C}^{n}$. Put in other words, if two distinct bounded domains have boundary orbit accumulation points, and if those boundary orbit accumulation points are both strongly pseudoconvex, then the two domains must be biholomorphic (since they are both biholomorphic to the ball).

One might more generally formulate this question

Suppose that $\Omega_{1}$ and $\Omega_{2}$ are two bounded domains in $\mathbb{C}^{n}$. Assume that $\Omega_{1}$ has boundary orbit accumulation point $P_{1}$ and $\Omega_{2}$ has boundary orbit accumulation point $P_{2}$. If $P_{1}$ and $P_{2}$ have the same Levi geometry, may we conclude that $\Omega_{1}$ is biholomorphic to $\Omega_{2}$ ?

I do not know the full answer to this question at this time. However, the following partial answer may be proved using known techniques: 
Proposition 3.1 Let $\Omega_{1}, \Omega_{2}$ be bounded domains in $\mathbb{C}^{n}$. Let $P_{1} \in \partial \Omega_{1}$ and $P_{2} \in \partial \Omega_{2}$ each be boundary orbit accumulation points. Assume that $\partial \Omega_{j}$ is smooth near $P_{j}, j=1,2$. Suppose that each $P_{j}$ is of finite type in the sense of Kohn/Catlin/D'Angelo and is also a peak point. Finally assume that there is a neighborhood $U_{1}$ of $P_{1}$ and a neighborhood $U_{2}$ of $P_{2}$ and a biholomorphic mapping

$$
\Phi: U_{1} \cap \Omega_{1} \rightarrow U_{2} \cap \Omega_{2}
$$

such that (i) $\Phi$ continues to a diffeomorphism of $\partial \Omega_{1} \cap U_{1}$ to $\partial \Omega_{2} \cap U_{2}$, (ii) $\Phi\left(P_{1}\right)=P_{2}$.

Then $\Omega_{1}$ is biholomorphic to $\Omega_{2}$.

Proof: Choose a point $X_{1} \in \Omega_{1}$ and automorphisms $\varphi_{j}$ of $\Omega_{1}$ so that $\varphi_{j}\left(X_{1}\right) \rightarrow P_{1}$. Likewise choose a point $X_{2} \in \Omega_{2}$ and automorphisms $\psi_{j}$ of $\Omega_{2}$ such that $\psi_{j}\left(X_{2}\right) \rightarrow P_{2}$. A standard argument (see [KRA1, Chapter 11) shows that, for any compact set $K \subseteq \Omega_{1}, \varphi_{j}(z)$ converges to $P_{1}$ uniformly for $z \in K$. A similar statement holds for $\Omega_{2}$.

Let $K$ be a large compact set inside $\Omega_{1}$. Choose $j$ so large that $\varphi_{j}(K) B U_{1} \cap$ $\Omega_{1}$. Likewise let $L$ be a large compact set inside $\Omega_{2}$. Choose $k$ so large that $\psi_{k}(L) B U_{2} \cap \Omega_{2}$. Let $\epsilon>0$ be small and set $U_{1}^{\epsilon}=\left\{z \in U_{1}: \operatorname{dist}\left(z,{ }^{c} U_{1}>\epsilon\right\}\right.$. Similarly set $U_{2}^{\epsilon}=\left\{z \in U_{2}: \operatorname{dist}\left(z,{ }^{c} U_{2}>\epsilon\right\}\right.$. By shrinking $\epsilon$ if necessary, we may assume that $\Phi\left(\varphi_{j}(K)\right) B U_{2}^{\epsilon}$. By enlarging $L$ if necessary, we may suppose that $\varphi_{k}(L) \supseteq U_{2}^{\epsilon}$.

Now consider $\left(\psi_{k}\right)^{-1} \circ \Phi \circ \varphi_{j}$. This will be a univalent holomorphic mapping that takes $K ß \Omega_{1}$ to $L$. And the mapping is invertible. We may similarly assume that the inverse mapping takes $L$ to $K$. The set of all such mappings, as $K$ exhausts $\Omega_{1}$ and $L$ exhausts $\Omega_{2}$ forms a normal family. And we may extract a convergent subsequence that converges to a biholomorphic mapping of $\Omega_{1}$ to $\Omega_{2}$. That is the result that we seek.

There are a number of different approaches to the classical Bun Wong/Rosay theorem. Useful references are [WON], [ROS], [KIK2], [KIK3], [KIK4], [GKK]. 


\section{Concluding Remarks}

In the paper [BEP1] and subsequent works, Bedford an Pinchuk prove results of the following type:

Theorem: Let $\Omega$ be a pseudoconconvex domain in $\mathbb{C}^{2}$ with real analytic boundary. Suppose that $\Omega$ has noncompact automorphism group. Then $\Omega$ must be biholomorphic to a complex ellipsoid of the form

$$
E_{m}=\left\{\left(z_{1}, z_{2}\right) \in \mathbb{C}^{2}:\left|z_{1}\right|^{2}+\left|z_{2}\right|^{2 m}<1\right\}
$$

for $m=1,2, \ldots$

In a private communication, David Catlin pointed out that the hypothesis of this theorem may be reduced to "finite type domain" in $\mathbb{C}^{2}$. In later papers Bedford and Pinchuk produced analogous results in $\mathbb{C}^{n}$.

But it must be pointed out that, in higher dimensions, we cannot hope for a conclusion as simple as "the domain must be a complex ellipsoid." For consider the domain

$$
\Omega^{*}=\left\{\left(z_{1}, z_{2}, z_{3}\right) \in \mathbb{C}^{3}:\left|z_{1}\right|^{2}+\left(\left|z_{2}\right|^{2}+\left|z_{3}\right|^{2}\right)^{2}<1\right\} .
$$

It has automorphisms of the form

$$
\Phi_{a}\left(z_{1}, z_{2}, z_{3}\right)=\left(\frac{z_{1}-a}{1-\bar{a} z_{1}}, \frac{\sqrt[4]{1-|a|^{2}} z_{2}}{\sqrt{1-\bar{a} z_{1}}}, \frac{\sqrt[4]{1-|a|^{2}} z_{3}}{\sqrt{1-\bar{a} z_{1}}}\right)
$$

for $a \in \mathbb{C},|a|<1$. If we let $a$ take the values $1-1 / j$ for $j=1,2, \ldots$ then we see immediately that $\Omega^{*}$ has noncompact automorphism group. And $\Omega^{*}$ is not an ellipsoid in the most direct sense. It has been conjectured by Catlin and others (see [KRA4] for the details) that the correct conclusion in higher dimensions is that the defining function of the domain should satisfy a certain homogeneity condition.

It has been noted that the Greene-Krantz conjecture asserts that a boundary orbit accumulation point must be of finite type. There is some evidence to support the conjecture - see, for instance, [KIM], [KIK1]. If it turns out to be true, then the Bedford/Pinchuk theorem cited above can be streamlined to say that a smoothly bounded domain in $\mathbb{C}^{2}$ with noncompact automorphism group must be an ellipsiond. 
It is certainly a matter of some interest to understand the nature of boundary orbit accumulation points. We know that they must be pseudoconvex, and the Greene-Krantz conjecture posits even more specific information about these points. Another subject of some study is boundary orbit accumulation sets - see, for instance, [ISK] and [KRA5]. Much more can in principle be said about these sets.

Automorphism groups are in some sense an invariant that is a substitute for the lack in several complex variables of a uniformization theorem or a Riemann mapping theorem. It is in our best interest to develop their properties so that they can be used effectively to study and classify domains up to biholomorphic equivalence.

We hope to study these matters further in future papers. 


\section{REFERENCES}

[ALA] G. Aladro, The comparability of the Kobayashi approach region and the admissible approach region, Illinois Jour. Math. 33(1989), 42-63.

[BEP1] E. Bedford and S. Pinchuk, Domains in $\mathbb{C}^{2}$ with noncompact group of automorphisms, Math. Sb. Nov. Ser. 135(1988), No. 2, 147-157.

[BEP2] E. Bedford and S. Pinchuk, Domains in $\mathbb{C}^{n+1}$, with non-compact automorphism group, Journal of Geometric Analysis 3(1991), 165-191.

[CAT] D. Catlin, Estimates of invariant metrics on pseudoconvex domains of dimension two, Math. Z. 200(1989), 429-466.

[GKK] H. Gaussier, K.-T. Kim, and S. G. Krantz, A note on the Wong-Rosay theorem in complex manifolds, Complex Var. Theory Appl. 47(2002), $761-768$.

[GRK1] R. E. Greene and S. G. Krantz, Invariants of Bergman geometry and results concerning the automorphism groups of domains in $\mathbb{C}^{n}$, Proceedings of the 1989 Conference in Cetraro (D. Struppa, ed.), 1991.

[HEL] S. Helgason, Differential Geometry and Symmetric Spaces, Academic Press, New York, 1962.

[ISK] A. Isaev and S. G. Krantz, On the boundary orbit accumulation set for a domain with non-compact automorphism group, Mich. Math. Jour. 43(1996), 611-617.

[KIM] K.-T. Kim, Domains in $\mathbb{C}^{n}$ with a piecewise Levi flat boundary which possess a noncompact automorphism group, Math. Ann. 292(1992), $575-586$. 
[KIK1] K.-T. Kim and S. G. Krantz, Complex scaling and domains with noncompact automorphism group, Illinois Journal of Math. 45(2001), $1273-1299$.

[KIK2] K.-T. Kim and S. G. Krantz, Some new results on domains in complex space with non-compact automorphism group, J. Math. Anal. Appl. 281(2003), 417-424.

[KIK3] K.-T. Kim and S. G. Krantz, A Kobayashi metric version of Bun Wong's theorem, Complex Var. Elliptic Equ. 54(2009), 355-369.

[KIK4] K.-T. Kim and S. G. Krantz, Characterization of the Hilbert ball by its automorphism group, Trans. Amer. Math. Soc. 354(2002), 2797-2818

[KRA1] S. G. Krantz, Function Theory of Several Complex Variables, $2^{\text {nd }}$ ed., American Mathematical Society, Providence, RI, 2001.

[KRA2] S. G. Krantz, Characterizations of smooth domains in $\mathbb{C}$ by their biholomorphic self maps, Am. Math. Monthly 90(1983), 555-557.

[KRA3] S. G. Krantz, On the boundary behavior of the Kobayashi metric, Rocky Mountain J. Math., 22(1992).

[KRA4] S. G. Krantz, Recent progress and future directions in several complex variables, in Complex Analysis Seminar, Springer Verlag Lecture Notes 1268(1987), 1-23.

[KRA5] S. G. Krantz, Topological/geometric properties of the orbit accumulation set, preprint.

[NAR] R. Narasimhan, Several Complex Variables, University of Chicago Press, Chicago, 1971. 
[ROS] J.-P. Rosay, Sur une characterization de la boule parmi les domains de $\mathbb{C}^{n}$ par son groupe d'automorphismes, Ann. Inst. Four. Grenoble XXIX(1979), 91-97.

[WON] B. Wong, Characterizations of the ball in $\mathbb{C}^{n}$ by its automorphism group, Invent. Math. 41(1977), 253-257.

Department of Mathematics

Washington University in St. Louis

St. Louis, Missouri 63130

sk@math. wustl . edu 